\title{
Research on the Application of Quality and Efficiency Evaluation in Small and Medium-sized Enterprises in Jilin Province
}

\author{
Qian Zhang ${ }^{1, a}$ \\ College of Humanities \& Sciences of Northeast Normal University , Changchun, Jilin Province \\ 130117, China
}

824974748@qq.com

Keywords: Small and medium-sized enterprises; Quality and efficiency; Evaluation; Management

\begin{abstract}
The concept of quality and efficiency evaluation is firstly proposed in judicial systems to assess judges' quality and efficiency of handling cases, thereby emphasizing process management. After being adopted by tax authorities, it is used to focus on the control of tax inspection. In my opinion, such concept should either be introduced in the study of the internal management processes of small and medium-sized enterprises, that is, conducting specific analysis of resources, costs, capabilities, etc. and figuring out the best balance point of quality and efficiency on the basis of the strategic schemes of the enterprises so that they can achieve greater value.
\end{abstract}

\section{An Overview of Quality and Efficiency Evaluation for Minor Enterprises}

Definition of Quality and Efficiency Evaluation for Minor Enterprises. Quality and efficiency of minor enterprises refers to the quality and efficiency of minor enterprises' management, namely, the degree of superiority and inferiority of work. Efficiency presents the correlation between the input or consumption and outcomes achieved by an enterprise over a period, which reflects the enterprise's cost and benefits. Quality and efficiency evaluation is able to give expression to the efficiency of different functional departments for an enterprise to measure the cost to management achievements and is a process where the work quality and management effect of the department evaluated are assessed and estimated. In the process, the quality and effect will be under consistent feedback and regulation, whose results will be put into use in a scientific and reasonable way. Owing to the flexibility in management and the uncertainty in future development, in terms of the institutional design, it requires minor enterprises to figure out the best quality-efficiency balance prior to conducting a comprehensive analysis and evaluation on resources, costs and capability according to corporate strategies and overall targets. Only in such way is the enterprise likely to maximize its gains. From the perspective of management quality and efficiency evaluation plays a role of not only the subset and key, but also the extension and expansion of performance evaluation. Thanks to its more standardized contents, clearer direction, more specific items and more quantified criteria, quality and efficiency evaluation appears to be purer and more accurate than performance assessment.

Futures of Quality and Efficiency Evaluation for Minor Enterprises. In order to understand the definition of quality and efficiency evaluation more deeply, it is necessary to give an analysis about the features of it. The characters of quality and efficiency evaluation are listed as below: Dynamics. The flexibility in management and the uncertainty in future development of minor enterprises cause the result that employees' performance will change at different periods. To be specific, these employees who perform better before may leave behind while those with worse performance probably gain improvement. Therefore, when it comes to the calculation of quality and efficiency appraisal cycle, it's suggested to take a full account of its dynamics and fix a proper cycle according to different types of evaluations on the basis of case by case so as to ensure that the organization enables to timely and fully control different levels of assessment based on the purpose of evaluation to reduce unnecessary management costs and reach an upper quality and efficiency level. In addition, under different circumstances, minor enterprises have distinct focuses on different items of quality and efficiency. Sometimes they put particular emphasis on efficiency, sometimes on 
effect, and other times they take all aspects into consideration. In any case, the development of minor enterprises is supposed to cognize and comprehend quality and efficiency with a view to institution and development. It is required to analyze and appraise the subject involved in quality and evaluation from different sides. According to Bred Rup, there should be three aspects: effectiveness, efficiency and revolution. Effectiveness means the degree of achieving goals; efficiency is enterprise's condition in resource input and output; revolution refers to enterprise's degree of readiness to cope with the changing market place. Interdependent on each other, the three aspects determine the competitive strength of enterprises. Multiple factors. Quality and efficiency evaluation for minor enterprises is influencing by dozens of factors, which involves both internal and external ones. Minor enterprises' internal factors cover organizational strategies, corporate culture social responsibility, organizational structure and manager's leadership, while external factors include economic environment, social environment, legal environment and the development of the trade. Under different situations, the effect of quality and efficiency evaluation appears to be different. With regard to analyzing the gap of quality and efficiency, only by the means of taking into account all the impacts from various factors can enterprises concentrate on key points and improve the level of quality and efficiency management.

\section{A Status Analysis of the Quality and Efficiency Evaluation for Jilin Minor Enterprises}

The progress of Jilin minor enterprises is inseparable from manpower introduction, development, training, utilization and other procedures. With time and technological improvement, it can become practicable to develop performance examination into quality and efficiency evaluation.

Defect in Institutional Design. Overall imbalance of the enterprise value chain. Enterprises put emphasis only on evaluating individual functional department but ignore the quality and efficiency balance of the value chain. The feature is commonly shared by almost all the minor enterprises in Jilin in the process of quality and efficiency evaluation. Enterprises create value through the corporation of all functional department and "group production". Provided that enterprises only focus on the appraisal of separate functional department, the relation among all of them is cut off from each other, thereby overlooking the measurement of value chain, which will generate a serious impact on enterprises' value creation on the whole. Setting appraisal indicators with financial figures. which does harm to the long-term development of enterprises. Secondly, it is easy for operators to overvalue and maintain the results achieved, which furthers their thoughts to seek quick success and instant benefits, and deeds for short-run speculation, and is adverse for enterprises to reach their long-term strategic object. Besides, figures from financial statement reflect the items in the past and fail to improve the internal operation of enterprises and the relationship between upstream and downstream consumers, which however, play a crucial part in enterprises' success under the era of knowledge economy.

Quality and Efficiency Evaluation Lacks Enforcement. Institutional fault. Institution fails when methods and procedures of quality and efficiency. evaluation are designed and chosen unreasonably and improperly. Currently, a number of minor enterprises apply blindly existing pattern and method of quality and efficiency evaluation from other successful enterprises, and some even outsource the whole human resource system, which is likely to make evaluated items disconnect from actual situation, lowering the effect of assessment results. Incomplete system for rewards a d punishment. Quality and efficiency management aims at two ultimate purposes. One is to provide basis for personnel decisions, and the other is focused on improving the quality of quality and efficiency management. The outcomes of quality and efficiency evaluation together with various information collected from the process of assessment can be applied into employee's vocational development planning, personnel decisions, training programs, remuneration as well as other corporate management. Imperfect system for rewards and punishment within enterprises causes the worst damage to positive and hardworking employees, who are exactly the mainstay of enterprises.

Information Communication Barriers in Quality and Efficiency Evaluation. Communication barriers will affect quality and efficiency presentation. Of all the enterprises 
investigated, it shows that insufficient early-stage preparations during the process of quality and efficiency evaluation are bad for making scientific and effective personnel decisions. It will have a serious influence on assessment effect if enterprises fail to make full preparations and well coordinate human resources before evaluation. It produces the same consequence if timely communication lacks during appraisal.

\section{A Project Design for the Quality and Efficiency Evaluation of Jilin Minor Enterprises}

To set Targets for quality and Efficiency Evaluation. Process for management by objectives. Management by objectives is regarded as a common method in quality and efficiency evaluation. Its main procedure is as below:

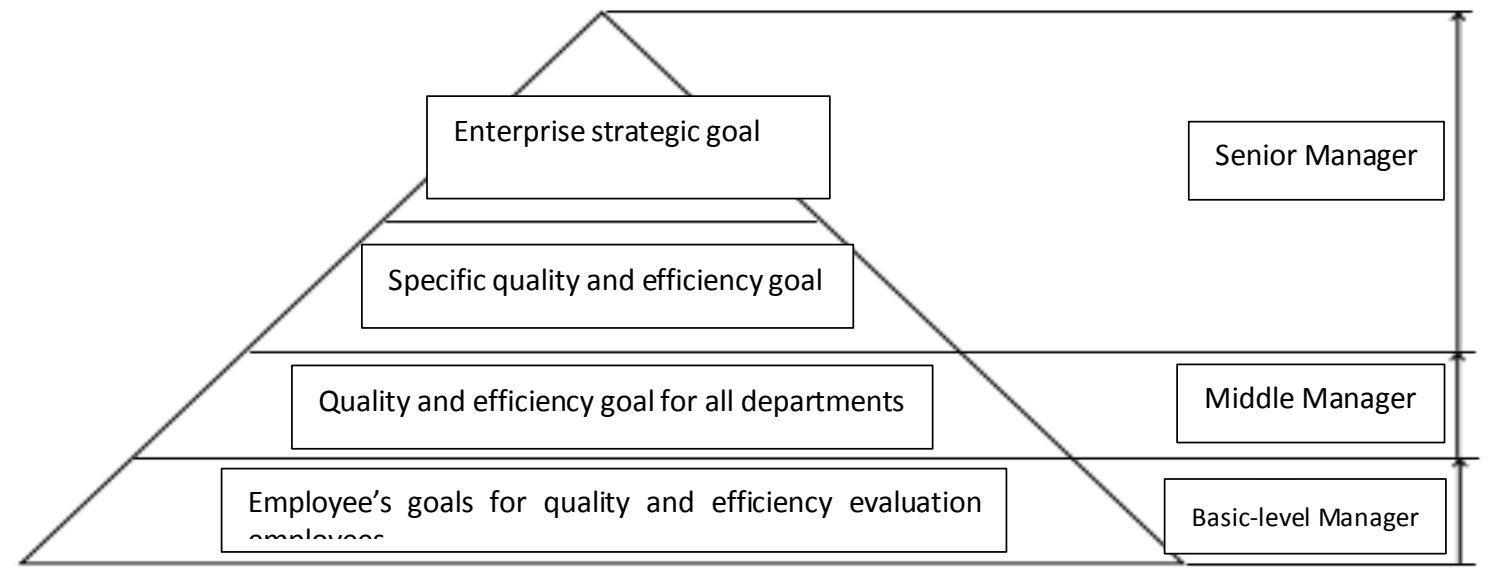

As a matter of fact, the objectives of quality and efficiency evaluation are determined through the discussion of superiors and subordinates so as to make different assessment targets for all levels. The above-mentioned objectives refer to the result to achieved and means and methods to be adopted in order to reach the result in organization and management. Goals setting is as below:

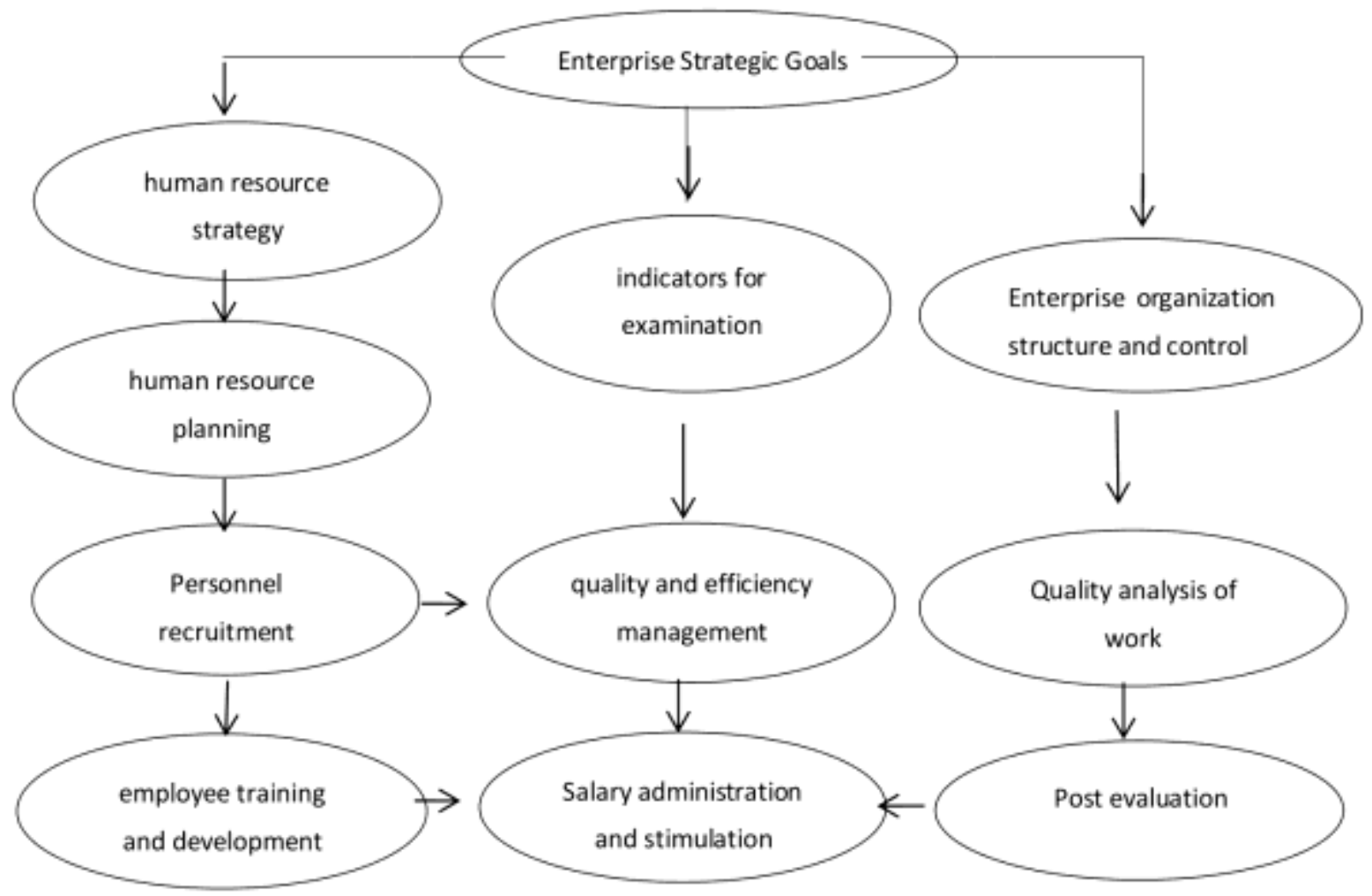


Steps to design quality and efficiency objectives. At first, enterprises are supposed to establish a strategic planning group. involving senior leaders, which takes charge of drawing up and giving description to enterprise's development vision. Afterwards, enterprise's strategic target will be fixed with the shared agreement among high-level leaders. The process to formulate objectives of quality and efficiency evaluation should be in line with following steps: It begins with the establishment of a strategic planning group with the participation of senior leaders. The group is mainly responsible for describing and making enterprise's future development vision and determines enterprise's strategic goals after leaders come to a census. Assuming that enterprise's strategic goals have been defined, it is required to work out an objective for quality and efficiency evaluation in combination with enterprise's annual plan; Enterprise's high-level leaders are supposed to form a leading group with people who manage different departments and put forward separate development goals for all departments. After that, they should specify the goals of quality and efficiency evaluation for their department. When setting quality and efficiency objectives for departments, managers need to pay attention to keep the quality and efficiency goals of their departments consistent with the general quality and efficiency objectives of the enterprise vertically, and synergistic to other functional departments horizontally; Administrators of different functional departments fully communicate with their staff concerning how to break down and reach their goals, forming different assessment criteria for individuals. During the process, leaders are required to make overall plans and coordinate each employee's work so that the department's goals can be achieved. To determine methods for quality and efficiency evaluation. As for the minor enterprises in Jilin, it is of special importance to make use of proper methods for quality and efficiency evaluation. Enterprises will apply different methods and focus on diverse aspects in various developing stages. At the initial period, enterprise's institutional and personnel structures are relatively simple, so quality and efficiency evaluation can be completed through qualitative assessment. Under such circumstances, it will cause unnecessary work if enterprises adopt complex quality and efficiency management system instead. To combine examination by immediate superiors with 360 Degree Appraisal.360 Degree Appraisal is also called all-rounded or multi-source evaluation,

Which is to collect evaluation data from different levels of staff and make a comprehensive appraisal to employees from various aspects. That is, the examinee and staff close to the examinee, including the examinee's superiors, peers, subordinates, upstream and downstream customers give anonymous comments on the examinee according to their information gained from all kinds of channels. Thereafter, professionals will deliver feedbacks to the examinee after a comparison between other people's comments and the examinee's self-assessment in order that the examinee is able to learn about his problems and make improvement. To apply the combination of quantitative and qualitative methods. Design of indicators for quality and efficiency evaluation must stick to objectivity and conciseness with the method of combining quantification with qualification. Based on post instructions, it is necessary to design reasonable indicators for quality and efficiency evaluation and give detailed explanations of the assessment criteria. Any method for quality and efficiency evaluation is equipped with corresponding evaluation indicators. On the basis of existing indicators for quality and efficiency evaluation, enterprises should scheme out a set of assessment criteria at the service of the actual situation about enterprise's characteristics. With regard to the minor enterprises in Jilin, generally, their administrative staff burden heavy tasks, so simple and clear assessment methods are easy to carry out and unlikely to cause impact on their major work.

Process to Design Quality and Efficiency Evaluation. Preparations for quality and efficiency plan cover a beginning from formulating. enterprise's strategic planning, a careful analysis of enterprise's present environment of competition as well as future position, and then it comes to fix the most effective implementation steps and integrate all resources for enterprise strategic goals; Communication of quality and efficiency plan serves as the core of the whole assessment process. Enterprise's management needs to fully communicate with employees so as to reach an agreement on employee's objective and plan in the process of evaluation. Throughout the communication, it is required to create a favorable atmosphere, specify the principles of communication, well design the communication process and bear a result for the communication; Formulation of criteria for quality 
and efficiency evaluation. Quality and efficiency criteria are supposed to reflect the fairness and objectivity of quality and efficiency evaluation and take shape based on work instead of staff. Position standards developed based on the features of post have to keep unified even though the same position is taken by lots of employees; Check and confirmation of quality and efficiency. When the quality and efficiency plan comes to an end, there should be following outcomes: employee's job aim is closely related to enterprise's overall objective; employee's duties are able to show the major tasks and responsibilities within the assessment process; the both sides involved in the evaluation reach a consensus about employee's main tasks, importance, standards of task completion and time limit; managers and stuff are clear about difficulties and obstacles they may be faced with and administrators are well aware of their support and assistance; there exists a file accepted by both sides through joint negotiation and with their signature. The quality and efficiency plan should be done in duplicate for enterprise's manager and employees respectively. The plan can be seen as not only employee's behavioral guidelines in future quality and efficiency cycle, but also a major basis for managers to conduct supervision, examination and appraisal; Feedback of quality and efficiency outcomes. Having evaluated employee's quality and efficiency, enterprise's managers are required to give feedback to and communicate with staff. Through interview, employees are expected to get involved into quality and efficiency evaluation to improve staff's satisfactory with enterprise management system effectively. It enables employees to know their immediate leader's understanding and views on their work, make constant improvement, enhance their working skills and dig out new development channels if leaders can figure out employee's problems and offer assistance. When the both sides of quality and efficiency evaluation agree on assessment results, they need to conduct an in-depth exploration into how to better quality and efficiency management goals and methods and make common efforts to amend quality and efficiency plan.

\section{Countermeasures and Suggestions for Jilin Minor Enterprises to Implement Quality and Efficiency Evaluation}

To Carry forward the Quality and Efficiency Evaluation of Jilin Minor Enterprises under Plans. Under the guidance of reform, Jilin minor enterprises should fully implement the new system of quality and efficiency evaluation, put into practice classified and hierarchical appraisal management, set goals for all departments and apply the "integration" of examination, supervision and feedback. Institutional framework changes towards "flattening". Enterprise's institutional construction is required to stand out rigidity and focus on people-oriented management philosophy with more specified evaluation indicators, clearer system of rewards and penalties, higher level enterprise cultural construction and special attention to the design of ideas.

To make the Plan for Quality and Efficiency Evaluation Practicable. The paper will take sales manager as an example to illustrate how Jilin minor enterprises should put quality and efficiency evaluation into practice. Contents of quality and efficiency evaluation for sales managers. As the business of sales has a strong orientation in objectives and techniques applied in quality and efficiency evaluation appear to be comparatively comprehensive, the focus of assessment indicators should be put on performance completion, loan collection percentage, comprehensive work capability, working discipline and attendance. Task completion. The evaluation for sales manager's performance is focused on how much sales targets he achieves during the business of enterprises, mainly involving performance to develop new customers, performance in sales volume, degree of rate protection, rate of loan collection, control of selling cost and budget five major performance indicators with the weight $40 \%, 20 \%, 20 \%, 10 \%$ and 10\% respectively (note: the percentage of weight is flexible and normally subject to the negotiation between decision-making level and examinee), among which assessment on sales performance takes up $70 \%$ of all. 


\section{Indicator design for Sales Performance Evaluation}

\begin{tabular}{|l|l|l|l|}
\hline \multicolumn{2}{|l|}{ Assessment criteria } & \multicolumn{2}{|l|}{} \\
\hline Assessment Indicator & Weight (\%) & $\begin{array}{l}\text { Percentage of } \\
\text { Completion (\%) }\end{array}$ & $\begin{array}{l}\text { Points for Single } \\
\text { Item (\%) }\end{array}$ \\
\hline $\begin{array}{l}\text { Process of new customer } \\
\text { development }\end{array}$ & $40 \%$ & 120 & 35 \\
\hline $\begin{array}{l}\text { Achievement of sales } \\
\text { volume }\end{array}$ & $20 \%$ & 100 & 20 \\
\hline Degree of rate protection & $20 \%$ & 99 & 18.4 \\
\hline Collection of loans & $10 \%$ & 88 & 11.56 \\
\hline $\begin{array}{l}\text { Budget control of selling } \\
\text { cost }\end{array}$ & $10 \%$ & 96 & 15.04 \\
\hline $\begin{array}{l}\text { Comprehensive } \\
\text { evaluation indicators }\end{array}$ & $100 \%$ & - & 100 \\
\hline
\end{tabular}

Evaluation of comprehensive capability. As for evaluation on sales manager's comprehensive capability, it needs sales director, purchasing manager, financial director and logistics manager to conduct an overall assessment from the aspect of sales manager's coordination ability, efficiency, personal potential and enthusiasm for work and then give regular evaluation outcomes. In accordance with the importance of all examinee's posts, enterprises are supposed to set various weights, work out the points and add into sales manager's total marks based on its percentage of 20\%; Evaluation on working discipline. Integrated administrative department will carry out a comprehensive assessment on sales manager's discipline and travelling employee's performance of following the regulations. Administrators are required to work out the points by deducting the part for breaking enterprise's rules according to sale's manager's general performance based on centesimal system, and put $15 \%$ of the total into sale's manager's overall evaluation. Such appraisal program should be applied to all posts where employee's performance can be directly measured.

To Complete a Scientific Mechanism for Quality and Efficiency Evaluation. To perfect working mechanism. The key for enterprises to formulate policy that reaches anticipated efficiency and effect lies in enterprise's executive capability. A sound enterprise leadership and working mechanism provide organizational guarantee for policy to run positively, which requires enterprises to build up a complete system and mechanism for enterprise's quality and efficiency management and make clear the leader in charge and staff in control to assign all duties to individuals. Meanwhile, enterprise's supervisory department needs to give full play to the function and make full use of supervision and put efforts to organizing and conducting quality and efficiency monitoring; To ensure the efficiency and effect of execution. Enterprise's institutional construction ensures that quality and efficiency management can be implemented smoothly and all work can be carried through as expected. Enterprise system generally belongs to post institution. Through a scientific analysis, enterprises break down all functions and objectives to separate post and specify the goals, mission and vision for all functional post. Moreover, enterprises should clarify the responsibilities and tasks for different positions and produce "Explanations for Position Statement" and "Measures for Quality and Efficiency Management" so as to supply standardized, constructive, binding and procedural basis and guide for normalizing quality and efficiency evaluation.

Feedback of Information Related to Jilin Minor Enterprise's Quality and Efficiency Evaluation. In quality and efficiency appraisal, enterprise's managers are regarded as the source of feedback, employees the object of feedback, and employee's performance and outcome of quality 
and efficiency assessment serve as feedback information throughout the quality and efficiency cycle. During the process, enterprises give feedback based on employee's actual work and behavior which is divided into correct, neutral and wrong behavior. For employee's correct behavior, positive feedback should be given. Employees are allowed to decide the result of feedback themselves when it comes to neutral behavior, and enterprises are supposed to send constructive opinions for incorrect deeds.

\section{Reference}

[1] Peter Drucker. The Effective Executive[M]. Mechanical Industry Press, 2005(01).

[2] Jon Werner. The Janus Performance System[M]. Electronic Industry Press, 2005(06).

[3] A Proposal on Formulating the 13th Five-Year Plan for National Economy and Society by the CPC Jilin Provincial Party Committee[J]. The New Long March, 2016(01).

[4] Shan Lijuan, Li Daqing, Li Qingman. Research on the Development of Liaoning High-tech Industry under the Background of Revitalization of Northeast China[J]. Journal of Bohai University, 2016(02).

[5] Lin Xinqi. Performance Management Manual[M]. China Labor and Social Security Press, 2006:89-217.

[6] Liu Weidong. On the Performance Management of Knowledge-based Employee[J]. Journal of Human Economic Management Cadre Institute, 2016(4).

[7] Hu Ying. Study on the Measures to Optimize the Service Structure of Jilin Province[J]. Social Science Forum.

[8] Luo Guogang. A Measurement System for the Dynamic Performance of Knowledge-based Employees[J]. Journal of Anhui University of Technology, 2015.6.

[9] An introduction to the Layout of Development Strategy and Opportunities for Investment and Cooperation of Changji Industry Innovation and development Demonstration Area. 2015.9.1

[10] Cai Yonghong, Lin Chongde. Status and Reflection on Performance Appraisal Research. Journal of Beijing Normal university, 2016(01).

[11]Li Songlin. Design and Implementation of Employee Performance Appraisal. Coal Mine Modernization, 2015(01).

[12] Peng L. Individual Choice and Reputation Distribution of Cooperative Behaviors among Heterogeneous Groups. Chaos, Solitons \& Fractals, 2015, 77: 39-46.

[13] Sun Haifa, Cheng Guangping, Liu Qianchuan. Performance Management[M]. Higher Education Press, 2015(02).

[14] Yu Weizhen. The Growth Mechanism on Collaboration-and-Innovation-Driven and Technology-based Minor Enterprises: Based on I-P-O Analysis Model[J]. Technology and Industry, 2016(02)

[15]Li Songqing, Zhou Jianyan, Zhang Qunzi. Evaluation and Research on the Ecological Innovation Level of Leading Enterprises in Agricultural Industrialization[J]. Journal of Human Agricultural University (Social Science Edition), 2016(01).

[16]Pu Dexiang. A Research on Knowledge-based Employee's Performance Based on Quality[J]. Guizhou Normal University.2017(03). 